\title{
Efficacy of transdermal glyceryl trinitrate in the treatment of chronic stable angina pectoris
}

\author{
M A JAMES, P R WALKER, M PAPOUCHADO, P R WILKINSON* \\ From the Department of Cardiology, Bristol Royal Infirmary, Bristol
}

SUMMARY A study was carried out to assess the efficacy of a new transdermal preparation of glyceryl trinitrate (Transiderm-Nitro 5) in the 24 hour prophylaxis of angina and to determine the duration of effect of a single patch application. Twelve men with chronic stable angina were studied in a randomised, placebo controlled, double blind trial. By serial treadmill exercise testing a therapeutic effect was shown at three hours; the exercise time to angina and to $1 \mathrm{~mm} \mathrm{ST} \mathrm{segment} \mathrm{depression} \mathrm{and}$ the total exercise time were all significantly increased. At 24,48 , and 72 hours, however, no therapeutic effect was observed. Recent studies have shown a similar lack of effect at 24 hours for various forms of transdermal delivery systems. It is suggested that this lack of effect is due to the rapid onset of tolerance probably as a result of the constancy of blood concentrations obtained by this method of administration.

Inhaled amyl nitrite was the first drug to be used for the relief of angina pectoris, and its successor, sublingual glyceryl trinitrate, remains one of the standard forms of treatment. ${ }^{1}$ The brevity of its action led to a search for longer acting preparations; thus various forms of oral nitrates became available and were shown to provide long term symptomatic benefit in ischaemic heart disease. ${ }^{2-4}$ Nevertheless, rapid hepatic metabolism led to difficulties in dosage titration, and confusion exists as to which metabolite is responsible for its major effects. ${ }^{5-7}$ Quite recently, nitrate creams applied to the skin have been shown to be efficacious since they avoid the problems of first pass hepatic metabolism..$^{8-10}$ These preparations are, however, messy to use, require frequent application, and do not permit accurate dosing. ${ }^{11}$ Thus the recent development of a new transdermal delivery system to release glyceryl trinitrate into the circulation at a constant rate for 24 hours ought to represent a notable advance. ${ }^{12-14}$ The transdermal system consists of a plaster patch containing a $25 \mathrm{mg}$ reservoir of glyceryl trinitrate paste. A semipermeable, rate limiting membrane on the inner surface is applied to the skin. The glyceryl trinitrate reservoir maintains a concentration gradient and the rate limiting membrane maintains a

Requests for reprints to Dr M A James, Department of Cardiology, Bristol Royal Infirmary, Bristol, Avon BS2 8HW.

^Present address: Ashford Hospital, Middlesex.

Accepted for publication 29 January 1985 constant delivery of $50 \mu \mathrm{g} / \mathrm{cm}^{2} / \mathrm{h}$. The absorption capacity of the skin is proportional to the depth and density of the capillary network; by applying the patch to the hairless skin of the trunk rather than to an extremity the rate of absorption is kept constant. ${ }^{15-17}$

This study was designed to test the efficacy and duration of action of a single glyceryl trinitrate patch.

\section{Patients and methods}

The trial was a randomised, double blind, crossover study. Twelve men (mean age 52.7 (range 33-66) years) completed the study. They were selected from a clinic population with chronic stable angina (median length of history 17 (range 4-56) months) and a positive exercise test (at least $1 \mathrm{~mm}$ horizontal or downsloping ST segment depression during treadmill exercise). Three of the 12 patients had concomitant medical conditions: one had hypercholesterolaemia, one hypertension, and one diabetes mellitus. Patients with unstable angina, recent myocardial infarction (within two months), valvar heart disease, heart failure, history of severe headaches, allergy to plaster, anaemia, Prinzmetal angina, and previous intolerance to nitrates were excluded. Antianginal agents other than beta adrenergic blockers and sublingual glyceryl trinitrate were withdrawn two weeks before the study. Nine patients were taking chronic beta blockade 

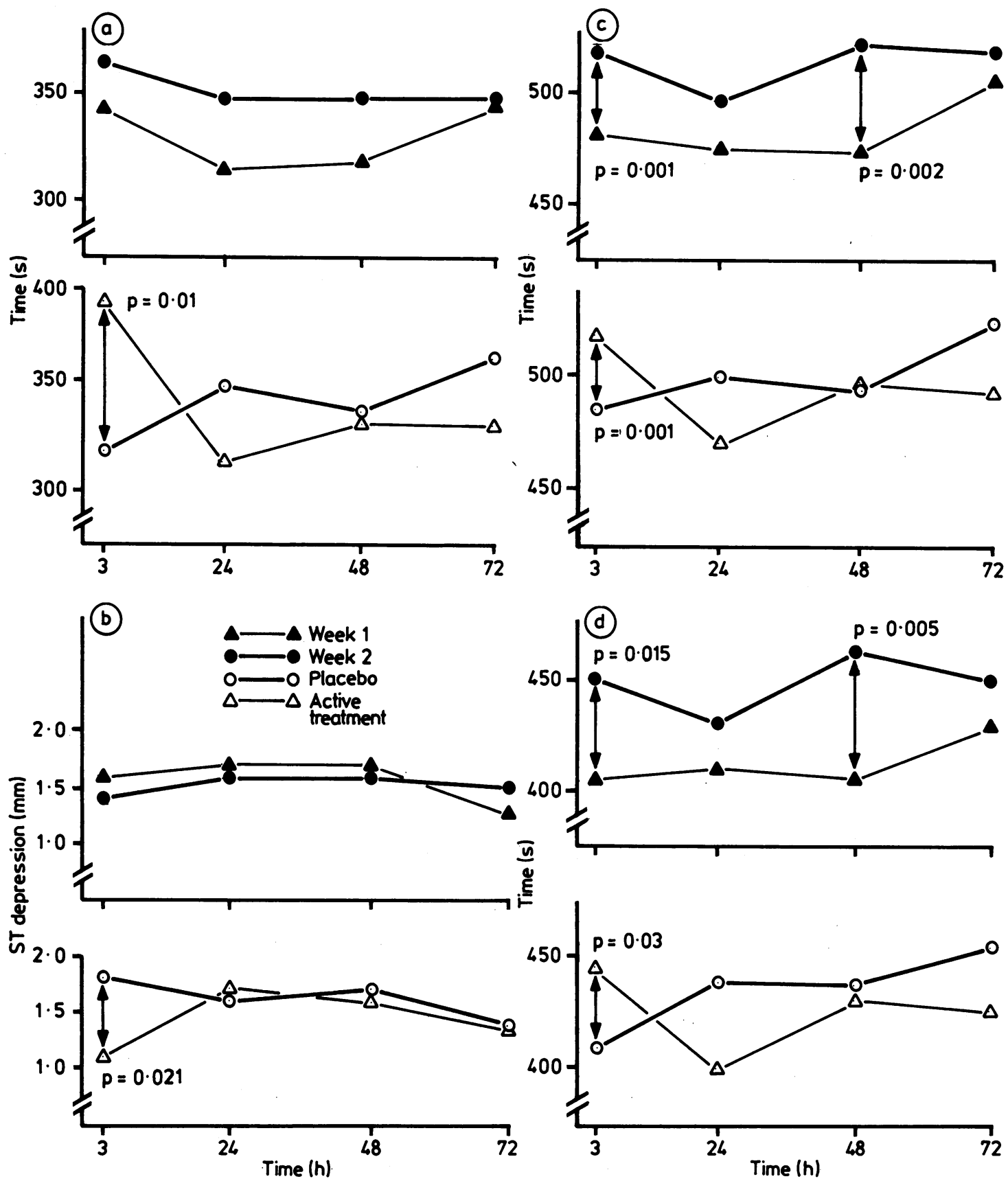

Figure Mean results of (a) time to $1 \mathrm{~mm} S T$ depression, (b) ST depression at minimum exercise, (c) total exercise time and (d) time to angina during exercise testing at $3,24,48$, and 72 hours of treatment in 12 patients receiving a glyceryl trinitrate patch or a placebo. The total values for week 1 and week 2 indicate the period effect or effect of training of serial exercise testing. The influence of training on the effects of treatment is allowed for in the calculation of the p values. Significant differences between two values are indicated by the bidirectional arrow. 
treatment, which was continued throughout the study.

The study consisted of a series of exercise tests performed over four successive days during two successive weeks with a three day break between each series of tests. Patients were randomised to receive either Transiderm-Nitro 5 or placebo treatment in their first week, receiving the other treatment in their second week. Exercise tests were performed at 3, 24, 48, and 72 hours after patch application using a modified Bruce protocol; the endpoint was a moderate, previously agreed level of angina. Twelve lead electrocardiograms were recorded at each minute of exercise and into recovery. For each patient the lead which showed the greatest ST segment depression during the first test was used for ST segment analysis on evey subsequent test.

The following variables were measured: $(a)$ time to $1 \mathrm{~mm}$ ST segment depression (at $60 \mathrm{~ms}$ after the J point); (b) time to angina; (c) total exercise time; and (d) ST segment depression at minimum exercise. Since a number of factors other than angina influence the total exercise time we decided to measure also the ST segment depression at a standardised point in the exercise protocol as well as at the maximum exercise time. This was termed the minimum exercise time and was obtained by measuring the amount of ST segment depression observed during the shortest exercise test and comparing this with the ST segment depression observed at the same minute of exercise in the other seven tests.

Statistical analysis was performed using the two period crossover technique described by Grizzle, 18 a $p$ value of $<0.05$ being considered to be significant. Results are expressed as mean (SD).

\section{Results}

Seven patients received their active treatment during the first week and five received placebo. The results of serial exercise testing are shown in the Figure. During active treatment there were significant increases at three hours in the time to angina $(446(100)$ vs 409 (130) $\mathrm{s}, \mathrm{p}=0.03$ ) and to $1 \mathrm{~mm} \mathrm{ST}$ segment depression $(384(100)$ vs $318(132) \mathrm{s}, \mathrm{p}=0.01)$ and in the total exercise time $(517(92)$ vs $484(118) \mathrm{s}, \mathrm{p}=0.01)$. The amount of ST segment depression at the minimum exercise time was also less at three hours $(1 \cdot 1(0.5)$ vs $1.8(1.0) \mathrm{mm}, p=0.02)$. These results take into account the training effect produced by serial exercise testing-that is, the subjects performed as a group better during the second week of the trial regardless of their randomisation order (Figure). At 24, 48, and 72 hours, however, no beneficial effects were seen during active treatment. During exercise testing at three hours after application of the nitrate patch the heart rate, systolic blood pressure, and double product (heart rate multiplied by systolic blood pressure) at the onset of angina, at $1 \mathrm{~mm}$ of ST segment depression, and at peak exercise were not significantly different from placebo.

The period of treatment ( 72 hours) was too short to assess its effects on the frequency of angina during daily activities, and therefore angina diary cards were not used; none the less, two patients expressed a preference for the active patch, two for the placebo, and eight had no preference. Eleven patients reported headache and lightheadedness during active treatment, which in four persisted for 24 hours, in three for 48 hours, and in three for 72 hours. Six patients also complained of these symptoms while taking placebo. In one patient severe headache and vomiting occurred during the first $\mathbf{2 4}$ hours of active treatment. In no patient was there a skin reaction.

\section{Discussion}

Serial exercise testing in these 12 patients showed a small but significant benefit from transdermal glyceryl trinitrate at three hours after patch application but not at 24,48 , or 72 hours. Recent studies of transdermal nitrates have produced conflicting results. Reichek et al were unable to show any improvement in exercise tolerance at 4,8 , or 24 hours after transdermal glyceryl trinitrate delivered at almost twice the dosage used in the present study, although at very high dosage (equivalent to more than $20 \mathrm{mg}$ of glyceryl trinitrate in 24 hours) a therapeutic effect was seen at four and eight but not at 24 hours. ${ }^{19}$ Parker $e t$ al, testing a patch containing $100 \mathrm{mg}$ of isosorbide dinitrate, found that exercise performance was improved at two, four, and eight hours but not at 24 hours. ${ }^{20}$ Naaes et al, evaluating a glyceryl trinitrate patch, found a significant improvement in exercise tolerance at 12-16 hours but did not test efficacy at 24 hours. ${ }^{21}$ Cerri et al tested two glyceryl trinitrate patches (Nitroderm TTS 5 and Nitroderm TTS 10) and found significant improvement in exercise tolerance at three and 24 hours, although there was no difference between the patches. ${ }^{22}$ On the basis of these studies, some scepticism that transdermal nitrate patches provide 24 hour prophylaxis in angina is justified. ${ }^{23} 24$

In our study, the absence of any therapeutic benefit at 24 hours and thereafter may have been due either to subtherapeutic plasma concentrations of glyceryl trinitrate or to the loss of end organ responsiveness-namely, tolerance. While the plasma concentrations obtained from a single TransidermNitro 5 patch are relatively low compared with those reported with other long acting nitrate preparations, ${ }^{16}$ the plasma concentrations have been shown to be con- 
stant for up to 28 hours. ${ }^{12-14}$ The plasma concentration is closely related to the surface area available for absorption ${ }^{15-17}$ and can be raised by increasing the size of the patch or applying more patches simultaneously. Thus in one study the glyceryl trinitrate concentration obtained from four patches applied simultaneously was more than three times greater than from a single patch.$^{17}$ Our data show, however, that a single application is sufficient to produce a significant, albeit small, therapeutic effect accompanied in almost all patients by headache. If it is assumed that diffusion has remained constant for 24 hours then the loss of therapelitic effect suggests the rapid development of tolerance. Muller $e t a l$ in their studies using transdermal glyceryl trinitrate noted an attenuation of the haemodynamic responses to the patch measured in the afternoon compared with the morning, although plasma concentrations of glyceryl trinitrate had not fallen. ${ }^{17}$ Tolerance has been found within 24 to $\mathbf{4 8}$ hours of commencing regular six hourly treatment with isosorbide dinitrate at both low and high dosage. ${ }^{25} 26$ In the recent study cited above, Parker et al were unable to show any benefit whatsoever from transdermal isosorbide dinitrate after 7-10 days of treatment, despite the initially significant therapeutic effect. ${ }^{20}$ There is evidence that any nitrate preparation which produces constant plasma concentrations and which therefore does not allow time for reversal of tolerance may quickly become ineffective. ${ }^{25}$ Parker et al have shown that the initial tolerance which occurs during chronic treatment may still be overcome by sublingual glyceryl trinitrate, ${ }^{20}$ again suggesting that haemodynamic responsiveness is dependent on rapid fluctuations in plasma concentrations. The mechanism of tolerance is unknown, but an opposing reflex neurohumoral vasoconstrictor response has been suggested. ${ }^{27}$

In other placebo controlled studies with long acting nitrates a higher rate-pressure product was achieved during active treatment. That this was not observed in our 12 patients may have been due to the attenuating effects of beta blockade in nine of them. Nevertheless, others have also found a lack of effect on the double product with nitrate patches used alone. 1920 Nitrates are effective in patients taking beta blockers, ${ }^{28}$ and the continued use of them throughout the study period should not have reduced the therapeutic effect of transdermal glyceryl trinitrate.

The stepwise improvement in exercise performance induced by training during a serial exercise training programme is well shown in this study by the observation that mean exercise time to angina and the duration of exercise were significantly greater during the second period compared with the first (Figure). Any consequent bias, which was in fact slightly in favour of placebo, was excluded by using the Grizzle two period changeover analysis in the calculation of the $p$ values. ${ }^{18}$

In conclusion, a single patch containing $25 \mathrm{mg}$ of glyceryl trinitrate produced a therapeutic effect on exercise performance at three hours after application, but this was not maintained at 24 hours or thereafter. It is likely that his loss of effect was due to the rapid development of tolerance, which may be related to the constant blood concentrations achieved by this delivery system. There is evidence that fluctuation of blood concentrations may be necessary to maintain haemodynamic responsiveness. ${ }^{20} 25$ A more significant benefit might be derived from a larger patch, but side effects could limit the efficacy of such a preparation, and even in a high dose the therapeutic effect seems to be lost by 24 hours. In view of the relatively high cost of this transdermal system compared with conventional, orally administered long acting nitrates, we suggest that further studies are necessary before this method of nitrate treatment be recommended for patients with chronic stable angina.

We thank Geigy Pharmaceuticals for supplying the active and placebo patches and for assistance with analysis of the data, Drs J Russell Rees, S C Jordan, and $J$ Vann Jones for allowing us to study patients under their care, and Mr L M Clarke and his staff for technical assistance.

\section{References}

1 Matthews MB. In: Julian DG, ed. Angina pectoris. Edinburgh, London, and New York: Churchill Livingstone, 1977: 5-6.

2 Thadani U, Fung H-L, Darke AC, Parker JO. Oral isosorbide dinitrate in the treatment of angina pectoris. Circulation 1980; 62: 491-502.

3 Markis JE, Gorlin R, Mills RM, Williams RA, Schweitzer P, Ransil BJ. Sustained effect of orally administered isosorbide dinitrate on exercise performance of patients with angina pectoris. Am $\mathcal{F}$ Cardiol 1979; 43: 265-71.

4 Lee G, Mason DT, Amsterdam EA, Miller RR, De Maria A. Anti-anginal efficacy of oral therapy with isosorbide dinitrate capsules. Chest 1978; 73: 327-32.

5 Needleman P, Lang S, Johnson EM Jr. Organic nitrates; relationship between biotransformation and rational angina pectoris therapy. 7 Pharmacol Exp Ther 1972; 181: $489-97$.

6 Bogaert MG, Rosseel MT, De Schaepdryver AF. Cardiovascular effects of glyceryldinitrates as compared to glyceryltrinitrate. Arch Int Pharmacodyn Ther 1968; 176: 458-60.

7 Wendt RL. Systemic and coronary vascular effects of the 2- and the 5-mononitrate esters of isosorbide. 7 Pharmacol Exp Ther 1972; 180: 732-42.

8 Meister SG, Engel TR, Guiha N, et al. Sustained haemodynamic action of nitroglycerin ointment. $\mathrm{Br}$ Heart f 1976; 38: 1031-6. 
9 Reichek N, Goldstein RE, Redwood DR, Epstein SE. Sustained effects of nitroglycerin ointment in patients with angina pectoris. Circulation 1974; 50: 348-52.

10 Parker JO, Augustine RJ, Burton JR, West RO, Armstrong PW. Effects of nitroglycerin ointment on the clinical and hemodynamic reponse to exercise. Am $\mathcal{F}$ Cardiol 1976; 38: 162-6.

11 Imhof PR, Ott B, Weiss A, Chu L-C, Chasseaud LF. Plasma concentrations and hemodynamic effects of percutaneously administered nitroglycerin and isosorbide dinitrate in healthy volunteers. In: Lichtlen PR, Engel H-M, Schrey A, Swan HJC, eds. Nitratres III. Cardiovascular Effects 3rd nitrate symposium Monte-Carlo, 1980. Berlin-Heidelberg-New York: Springer, 1981: 66-74.

12 Imhof P. Data on file. Geigy Pharmaceuticals, Horsham, West Sussex RH12 4AB.

13. Shaw JE. Transdermal therapeutic systems. In: Elsdon-Dew RW, Birdwood GFB, eds. Transdermal nitrates in ischaemic heart disease. International congress and symposium series, London Royal Society of Medicine 1983; 59: 33-5.

14 Place VA. Data on file ALZA Corporation, Pala Alto, California 94304, USA.

15 Scheuplein RJ, Blank IH. Permeability of the skin. Physiol Rev 1971; 51: 702-47.

16 Data on file, Geigy Pharmaceuticals, Horsham, West Sussex, RH12 4AB.

17 Muller P, Imhof PR, Burkart F, Chu L-C, Gérardin A. Human pharmacological studies of a new transdermal system containing nitroglycerin. Eur $\mathcal{F}$ Clin Pharmacol 1982; 22: 473-80.

18 Grizzle JE. The two period changeover design and its use in clinical trials. Biometrics $1965 ; 21: 467-80$. (correction: Biometrics 1974; 30: 727.

19 Reichek N, Priest C, Zimrin D, Chandler T, St John Sutton M. Antianginal effects of nitroglycerin patches. Am $\mathcal{F}$ Cardiol 1984; 54: 1-7.

20 Parker JO, VanKoughnett KA, Fung H-L. Transdermal isosorbide dinitrate in angina pectoris: effect of acute and sustained therapy. Am F Cardiol 1984; 54: 8-13.

21 Naafs MAB, De Boer AC, Koster RW. Klazen CW, Dunning AJ. Exercise capacity with transdermal nitroglycerin in patients with stable angina pectoris. Eur Heart f 1984; 5: 705-9.

22 Cerri B, Grasso F, Cefis M, Pollavini G. Comparative evaluation of the effect of two doses of nitroderm TTS on exercise-related parameters in patients with angina pectoris. Eur Heart f 1984; 5: 710-15.

23 Abrams J. The brief saga of transdermal nitroglycerin discs: paradise lost. Am f Cardiol 1984; 54: $220-4$.

24 Balcon R. Comment on papers by Naaes et al, and Cerri et al. Eur Heart f 1984; 5: 715.

25 Parker JO, Fung H-L, Ruggirello D, Stone JA. Tolerance to isosorbide dinitrate, rate of development and reversal. Circulation 1983; 68: 1074-80.

26 Thadani U, Manyari D, Parker JO, Fung H-L. Tolerance to the circulatory effects of oral isosorbide dinitrate. Circulation 1980; 61: 526-35.

27 Packer M, Meller J, Medina N, Yushak M, Gorlin R. Determinants of drug response in severe chronic heart failure. 1 Activation of vasoconstrictor forces during vasodilator therapy. Circulation 1981; 64: 506-14.

28 Baxter RH, Lennox IM. Increased exercise tolerance with nitrates in beta-blockaded patients with angina. $\mathrm{Br}$ Med $\mathcal{F}$ 1977; ii: 550-2. 